\title{
Germanica
}

\section{Le Kaffeehaus, lieu de modernité}

Das Kaffeehaus als Ort der Moderne

\section{Béatrice Gonzales-Vangell}

\section{CpenEdition}

Journals

Édition électronique

URL : http://journals.openedition.org/germanica/586

DOI : $10.4000 /$ germanica.586

ISSN : 2107-0784

Éditeur

Université de Lille

\section{Édition imprimée}

Date de publication : 1 décembre 2008

Pagination : 173-182

ISBN : 978-2-913857-22-6

ISSN : 0984-2632

\section{Référence électronique}

Béatrice Gonzales-Vangell, «Le Kaffeehaus, lieu de modernité », Germanica [En ligne], 43 | 2008, mis en ligne le 01 décembre 2010, consulté le 06 octobre 2020. URL : http://journals.openedition.org/ germanica/586 ; DOI : https://doi.org/10.4000/germanica.586

Ce document a été généré automatiquement le 6 octobre 2020.

(C) Tous droits réservés 


\title{
Le Kaffeehaus, lieu de modernité
}

\author{
Das Kaffeehaus als Ort der Moderne
}

Béatrice Gonzales-Vangell

1 L'introduction d'une denrée étrangère à la culture traditionnelle et l'intérêt qu'elle suscite vont permettre d'édifier des bâtiments voués à la démocratisation du café. Certains deviendront des institutions légendaires et entreront dans la tradition. Le café ou Kaffeehaus, n'est-ce pas la modernité ${ }^{1}$ ? L'allusion au café dans la littérature n'est pas anecdotique car elle véhicule une certaine idée du monde et du sujet qui l'habite. Son entrée dans la littérature sera évoquée ici dans les écrits d'Arthur Schnitzler, puis dans ceux de trois écrivains viennois de la fin $\mathrm{du} \mathrm{xx}^{\mathrm{e}}$ siècle: Robert Schindel, poète et romancier, né en 1944, Robert Menasse, né en 1954, philosophe, germaniste et romancier, et Doron Rabinovici, historien, essayiste et romancier né en 1961. Dans un essai, Doron Rabinovici déclare que « sans les cafés la littérature serait sans abri ». Quel est le sens de cette déclaration? Que nous apporte-elle comme éclairage sur la lecture de la modernité viennoise aujourd'hui ? Si l'on retient de la modernité l'idée d'un mouvement inachevé, l'instant d'irruption, d'un déchirement de l'identité entre tradition et renouveau, où espace et temps sont indissolubles, ne peut-on pas voir dans le traitement littéraire du lieu café ce que Bakhtin appelait un chronotope ${ }^{2}$ ?

2 Selon une fausse légende ${ }^{3}$, les cafés apparaissent à Vienne pendant le siège des armées ottomanes en 1683. La légende peut être fausse, l'idée subsiste d'une rencontre fructueuse entre Orient et Occident ${ }^{4}$. Un an plus tard, un premier établissement est inauguré, alors qu'il en existe déjà à Londres depuis 1652 et à Paris depuis 1689. En effet, le Procope, symbole d'une autre modernité, celle de Diderot et d'Alembert y a ouvert ses portes. Toutes les capitales européennes vont bientôt saluer l'ouverture de cafés : en 1678 à Hambourg, en 1681 à Venise, à Prague en 1712, à Budapest en 1848 et 1867. Le café est sans conteste un phénomène qui surgit dans le contexte européen à un moment qualifié provisoirement de moment de rupture.

$3 \mathrm{Au}$ cours du XIXe siècle, les villes européennes changeaient de visage. Vienne ne fit pas exception. À la démolition des anciennes fortifications en 1858 succéda la construction de grands axes. Les édifices qui bordent le Ring (boulevard circulaire) seront caractérisés par leur gigantisme et l'historicisme. La destruction des structures 
urbaines médiévales, dont la rue étroite et sinueuse était l'une des caractéristiques, allait fondamentalement transformer le rapport à la ville devenue la proie d'architectes qui servent la raison d'état. À Vienne, par exemple, il s'agit d'asseoir le prestige de la double monarchie pour écraser la contestation grandissante dont son autorité fait l'objet. À Paris, vers 1860, les grands boulevards furent percés afin de maittriser d'éventuels soulèvements populaires ${ }^{5}$. Le café devint alors ce lieu où, pour parodier la phrase de Baudelaire, « on est hors de chez soi et pourtant on se sent chez soi, on voit le monde, on est au centre du monde et on reste caché au monde $»^{6}$ ou, comme l'écrit Claudio Magris, « on se trouve à la fois enfermé et à l'air libre, dans l'illusion des deux » 7. Au café, lieu du dedans et du dehors, «la boisson est ce qui compte le moins ", dira Edmund Wengraf ${ }^{8}$. En revanche, le café, dans lequel une certaine intimité fut recréée, faisait oublier le visage impersonnel de la grandiloquence architecturale et, en ce sens, a un rôle social.

4 À Vienne, le progrès technique ne servit pas seulement à réaffirmer la grandeur et la puissance de l'empire. À partir de 1897, la Sécession donna un élan inédit. Le Café Museum conçu par Adolf Loos en 1899 était le fruit d'une réflexion esthétique : il s'agit de débarrasser l'architecture de la pesanteur esthétique du style néo-gothique et de l'historicisme et de tendre non pas vers une reconstruction du passé mais vers la création d'un nouveau langage. Dépassant le motif ornemental - même le motif géométrique et rectiligne de l'Art Nouveau viennois qui était déjà en soi une révolution - Loos parvint à des formes épurées et claires. Dans son architecture, le café épouse l'air du temps et sera immortalisé dans la littérature : par exemple chez Walter Serner, Elias Canetti, Joseph Roth, Hermann Broch, Stefan Zweig, Arthur Schnitzler ou, encore en 1992, dans les écrits de Robert Schindel, écrivain viennois né en $1944^{9}$.

5 Au-delà de ces répercussions topographiques, la transformation des villes eut pour conséquence la naissance d'un nouveau mode de vie. " Nouveau », " neuf », « jeune » sont les adjectifs qui définissent l'époque dont le nouveau style sera proclamé au café. Lieu privilégié des permutations, il sera un refuge pour les artistes peintres refusés au salon de Paris, une seconde résidence ${ }^{10}$ pour Else Lasker-Schüler, Herwarth Walden et son cercle ${ }^{11}$ à Berlin. Il sera à Vienne un lieu d'élection pour les hommes de Lettres qui se mêlaient aux laissés pour compte et aux jeunes diplomés au chômage ${ }^{12}$. Dès lors, on peut se demander si le café, scène culturelle comme l'avait été le salon littéraire au XVIII ${ }^{\mathrm{e}}$ siècle, ne marque pas le passage vers la modernité. À mode de vie nouveau, moyens d'expression inédits.

6 Les journaux à gros tirage et les gazettes apparurent, les cafés servaient souvent d'annexes aux rédactions des grands quotidiens et des revues influentes. Les nouvellistes et les écrivains participent à leur élaboration et à leur rédaction. Très rapidement, un lien se noue entre presse et littérature.

7 À Venise, Vienne, Paris, Berlin, Londres et plus tard Prague ${ }^{13}$, pour ne citer que ces villes, les habitués lisaient au café les journaux de toute l'Europe ${ }^{14}$ et s'entretenaient de politique. Le café, situé à la frontière de l'espace public, la rue, et de l'espace privé, l'appartement, était autour de 1900 l'épicentre de la vie intellectuelle en Europe.

8 Le café n'est pas seulement lieu de réception journalistique : de nombreux écrivains y créèrent des revues et c'est au café que l'on théorisa la modernité. Ne retenons parmi les innombrables parutions que Die moderne Dichtung d'Eduard Michel Kafka en 1890 dans laquelle furent édités les travaux du groupe Jung Wien. C'est au Café Griensteidl ${ }^{15}$, fondé en 1844 sur la Michaeler Platz qu'autour de Hermann Bahr se réunissaient de façon 
informelle les écrivains. Ils ne formaient ni une école, ni un groupe homogène. Bahr résuma ce qui les caractérisait : «La jeune Autriche ne copie pas le prototype berlinois, et n'a rien avoir avec l'exemple parisien, elle n'est pas révolutionnaire, elle n'est pas naturaliste. Qu'est-elle donc? $»^{16}$.

La jeune Vienne, dans son refus du réalisme et du naturalisme, parce qu'il ne proposait pas la rupture qu'elle souhaitait introduire, s'inspirait du symbolisme et de l'impressionnisme. C'était un choix conscient porteur de la modernité vers laquelle elle veut tendre. Lorsque le Griensteidl ferma ses portes, Karl Kraus se détacha du groupe constitué, entre autres, d'Arthur Schnitzler, Leopold Adrian, Richard Beer-Hofmann et Hugo von Hofmannsthal qui se retrouvèrent au Café Central. Leur attachement à la monarchie, leur ouverture à de nouvelles influences (Wilde, Barrès, Ibsen, Baudelaire, Mallarmé...), à toutes les formes d'art et leur recherche de nouvelles formes littéraires les rapprochaient. Chacun d'eux eut une lecture particulière du tournant du siècle dont ils furent les acteurs et les témoins attentifs. D'autres personnalités contribuèrent à fonder la légende du café littéraire : Alfred Polgar, Felix Salten ou Peter Altenberg par exemple qui conçut une "Théorie du café Central» dans laquelle le café était la solution à tous les soucis de la vie quotidienne. Stefan Zweig, lui, y voyait « la meilleure école pour tout ce qui était nouveau $»^{17}$.

Il n'en est pas autrement pour le jeune Arthur Schnitzler comme en témoigne le récit de sa jeunesse à Vienne. Alors qu'un ami de son père peu convaincu de son talent tenta de le dissuader de s'engager dans la voie littéraire, Arthur Schnitzler ne se laissa pas décourager et après avoir entendu l'avis défavorable, il choisit le Café Central pour se remettre à écrire : "Car à peine levé de table, je me rendis au Café Central et dans un coin à la lumière artificielle je commençai à écrire une autre pièce avec le titre: 'Modernes Jugendleben' ${ }^{18}$.

11 Schnitzler ajouta que, plus tard, certains aspects de cette esquisse sont réapparus dans Anatole. Dans ce court passage, le café viennois devient un espace où se révéle la vocation de l'écrivain. Ici, le passage au café devient moment d'inspiration, celui des muses, moment où se réalise le destin, celui du fatum. Il est point charnière entre destinées individuelles et mouvements artistiques collectifs, point de rencontre entre les diverses disciplines, espace polivalent et polymorphe où s'abolissent les frontières entre les genres, se nouent les dialogues et se tissent les correspondances.

Le café apparaît à la fois comme le cadre et le réceptacle qui permit la rencontre de la littérature et de la musique, de la science, de l'architecture et de la peinture comme si le café contenait et libérait les idées qui étaient brassées entre ses murs. Et la psychanalyse offrait une nouvelle dimension, Elias Canetti parlera plus tard de l'omniprésence des conversations sur Freud :

D'une certaine manière, chacun trouvait son Ædipe, et à la fin, tout le monde était assis là, coupable, tous de potentiels amants de leur mère et assassins de leur père, tous enveloppés d'un brouillard des noms mythiques, rois secrets de Thèbes ${ }^{19}$.

La nouveauté des modernes résidait, en effet, pour parler avec J.-Y. Masson, «dans l'exploration d'un monde intérieur ${ }^{20}$ dont le contrepoint était le sentiment de la dissolution des valeurs, sujet traité plus tard dans les romans des années vingt et trente. Le monde bercé, ordonné par la tradition s'évanouit comme en atteste ce court passage tiré d'une lettre de Hofmannsthal adressée à Beer-Hofmann en $1893^{21}$ :

À demi nostalgique d'une époque désormais révolue, j'étais

Comme un marcheur portant en bandoulière un luth 
Qui bat, à chaque pas, contre son épaule,

Et où le vent vient se prendre avec tant d'ardeur impatiente

Qu'il a le cœur oppressé comme par un souvenir ${ }^{22}$.

14 1900 correspondait à une crise de la subjectivité, leitmotiv de l'époque que la psychanalyse ${ }^{24}$ mit en évidence. Mais les prémisses de la découverte de la psychanalyse sont déjà présentes dans l'acte «La question au destin ${ }^{25} \mathrm{du}$ cycle Anatole de Schnitzler représenté pour la première fois en 1896 à Leipzig ${ }^{26}$. Le personnage féminin s'y laisse hypnoser par son amant qui veut s'assurer de sa fidélité. Même si le sujet hypnotisé ne présente pas de symptôme pathologique, même si, dans cette pièce, l'hypnose n'est pas une méthode thérapeutique et que la conclusion est ironique, les femmes mentent même quand elles sont dans un état d'hypnose, cette scène met en évidence la division de la conscience ${ }^{27}$ et donc l'éclatement du moi comme phénomène. Charcot et Freud ne semblent pas loin, Ernst Mach non plus. À partir de Die Analyse der Empfindungen (L'Analyse des sensations) de Mach publiée en $1886^{28}$, Otto Weininger constate, en 1903, l'effritement " des notions classiques de l'identité et pour appeler à sa reconstruction " 29. Ernst Mach, professeur de philosophie à Vienne depuis 1895, affirmait contre Kant que la chose en soi n'existait pas. Mach « exprime l'exigence d'abolir toute séparation entre le physique et le mental, l'objet et le sujet ${ }^{30}$. Ce n'est pas ce que nous appelons «les choses (les objets, les corps) mais bien les couleurs, les tons, les pressions, les espaces, les durées, ce que nous appelons les sensations, qui sont les véritables éléments du monde ». Hermann Bahr reprit la formule de Mach : « Das unrettbare Ich » (le moi irrécupérable) et la rendit populaire ; en 1908, Robert Musil fit dans sa thèse ${ }^{31}$ une critique positive de Mach.

Lieutenant Gustl, la nouvelle de Schnitzler. C'est la première fois dans la littérature de langue allemande qu'une nouvelle est entièrement composée d'un monologue intérieur. Toute la scène se déroule la nuit après une représentation à l'opéra où un boulanger a insulté le lieutenant Gustl. Pour sauver son honneur, il devrait se livrer à un duel. Mais se battre en duel contre un boulanger n'est pas pensable, le suicide est l'issue à laquelle tente de se résoudre Gustl sans y parvenir. Non pas la perte de son honneur mais la prise de conscience qu'il n'aurait pas le courage de se livrer à un duel le désespère. Il se réfugie dans un café où le garçon de café lui annonce au petit matin la mort subite du boulanger. Personne ne sait que son honneur a été sali. Le monologue est composé de questions qu'il s'adresse à lui-même, de bribes de phrases, d'exclamations et de perpétuelles tentatives d'auto-persuasion. La technique du monologue intérieur met en évidence la dichotomie entre, d'une part, le rôle que lui imposent la société, les conventions, son rang et, d'autre part, ses sentiments, ses peurs et ses incertitudes. L'honneur est vide de sens aux yeux de celui qui pense devoir affronter la mort. Finalement, le lieutenant n'est qu'un homme sans foi ni loi qui parvient à peine à se maîtriser lorsqu'il apprend la mort accidentelle du boulanger.

Le lieutenant choisit le café pour passer ce qu'il croit être les derniers moments de sa vie et l'auteur choisit le café pour dévoiler la vraie nature du protagoniste qui incarne l'armée de l'Autriche-Hongrie. Le garçon de café est le messager du destin, le café le refuge pour le moi déchiré par ses propres contradictions et finalement le lieu où tombent les masques. 
17 Les auteurs de la modernité étaient dotés d'une préscience qui leur fit entrevoir l'imminence des catastrophes à venir. Robert Schindel, Robert Menasse et Doron Rabinovici, écrivains viennois de la deuxième génération après la Shoah, ne jettent pas un regard passéiste sur la modernité, ils puisent à sa source tout en inscrivant dans l'avenir de leurs écrits la fracture de l'Histoire. Ils se réapproprient la langue allemande en réhabilitant sa fonction éthique (grâce à divers procédés qu'il serait trop long d'expliciter $)^{32}$. La continuité littéraire dans laquelle ils se placent est celle des hommes de lettres qui fréquentaient les cafés. Les biographies et les parcours respectifs de ces écrivains divergent, leur style et les thèmes qu'ils abordent les différencient certes, cependant le regard qu'ils portent sur la tradition les rapproche jusqu'à faire d'eux non pas une école mais un mouvement inédit sous bien des aspects. Seul le plus prégnant dans le cadre de notre étude sera évoqué.

Loin de se lancer dans une reconstruction historique du café, les trois écrivains le revisitent. L'allusion au café est programme dans l'œuvre de Robert Schindel, il place ses premiers écrits de fiction dans le café viennois : «Vers six heures moins le quart, l'atmosphère vespérale envahit aussi le café $»^{33}$.

19 Cette phrase récurrente dans la nouvelle semble être la métaphore de l'histoire, le café en est le microcosme. Même lorsqu'il est à Berlin, Schindel se rend inévitablement au Café Einstein, seul représentant digne du Kaffeehaus, du café viennois, comme dans son poème Immernie 10, au café Einstein ${ }^{34}$. Pour rendre justice à la force symbolique du café dans les écrits de Schindel, ces exemples sont maigres. On retiendra cependant que la visite au café est un rituel fondateur d'identité : identité d'écrivain, identité juive.

Dans son roman, Suche nach M., publié en 1997, le narrateur de Doron Rabinovici, évoque le café au début du premier chapitre: "Le café, son café préféré, avait été modifié dix ans après la guerre $»^{35}$.

21 La description précise qui suit de ce que le café fut avant la guerre sert à créer un décor aux personnages incarnant les rescapés de la Shoah qui, bon gré mal gré, sont revenus à Vienne. Le café fait figure de lieu transitoire où les personnages, notamment celui du père, originaire de Pologne, va pouvoir, après l'avoir falsifié pour échapper à la persécution, prononcer son nom pendant un rêve où il se trouve au café:

«Je suis Jakob Scheinowitz. Vous entendez? Docteurdocteur Jakob Scheinowitz!» 36.

22 Le café, Kaffeehaus, devient topos identitaire. C'est en ce sens que dans le roman de Robert Schindel, Gebürtig, (Le mur de verre), publié en 1992, Daniel, blessé après un accident de la circulation, déclare : «Un juif fait partie du Kaffeehaus, ai-je dit en interrompant son flot de paroles, je ne savais pas pourquoi cette idée m'était passée par la tête $»^{37}$.

Le juif reprend place dans une ville que Hugo Bettauer avait appelée en 1922 «la ville sans juifs ${ }^{38}$. Le souvenir du café du début du $x x^{\mathrm{e}}$ siècle est évoqué à petites touches, il n'apparaît que de façon plus présente. Dans Chassés de l'enfer ${ }^{39}$, Robert Menasse insère une anecdote intitulée "Métier : habitué de café, une histoire vraie », publiée en 1997. Après 1945, son grand-père, représentant de café, était pour ainsi dire un habitué professionnel. À partir de cette anecdote, l'auteur se livre à une variation stylistique autour des cafés et de la mémoire. Viktor, le personnage principal du roman, se demande comment ses grands-parents ont survécu à la Shoah. Le grand-père de Viktor 
évite à plusieurs reprises de répondre. Il s'adresse plutôt à sa femme et l'interroge : « Du reste Dolly, sais-tu, qui j'ai rencontré cet après-midi au Café Monopol ? $»^{40}$.

En évoquant le café Monopol plutôt que les camps, il instaure un lien entre passé et présent et fait du café un lieu positif de la mémoire. Chez Robert Menasse, le café devient l'emblème d'une époque et celle d'un art de vivre qui subsista pendant et après l'exil. On peut presque affirmer au-delà de l'exil. Le passage de Sinnliche Gewißheit le montre : le personnage féminin qui parle est originaire de Vienne. Elle vit depuis les années trente au Brésil. Au cimetière où elle enterre sa fille Judith, elle dit à Roman pourquoi elle n'est jamais revenue à Vienne :

Non. Pourquoi? Mon cher Monsieur le Docteur, vous savez bien comment cela se passe. Quand un Viennois a son café habituel et que ce café-là ferme ses portes pour une raison quelconque, alors il change juste de café. Et quand des années après, le premier café est rouvert par un nouveau propriétaire, le véritable Viennois n'y retourne pas mais il reste dans le café dans lequel il a passé les dernières années, où les garçons de café le connaissent déjà, où sont aussi assis ses amis, où il connaît déjà les autres habitués. Non vraiment, Monsieur, le Docteur, nous n'avons vraiment jamais pensé revenir, pour nous, il était toujours évident que nous resterions désormais dans ce café-là... ${ }^{41}$

Le café devient synonyme de pays. S'exiler équivaut à changer de café. L'habitude d'un lieu remplace l'amour de la patrie. Si l'on considère la relative banalité du lieu (le Kaffeehaus) et de ce qui s'y passe (commander une boisson à un homme dont le métier est d'apporter cette boisson), changer de café n'a rien de dramatique. Pourtant, la simplicité des faits relatés ainsi que le dénuement du style sont des euphémismes pour exprimer la tragédie de l'exil. L'allusion au café est, chez les trois écrivains, également le lieu de la fragmentation du sujet écartelé entre deux mondes, comme dans cette incise dans le roman de Robert Schindel où l'alter ego du narrateur déclare : « Du reste, je suis quelque peu dispersé dans le café $»^{42}$.

26 Le je n'est pas une entité homogène, il désigne un personnage, figure incarnant l'éclatement du moi. Cet éclatement, si paradoxal que cela paraisse, permet aux écrivains de se placer dans une continuité littéraire. Ainsi le sujet de la fin du $\mathrm{xx}^{\mathrm{e}}$ siècle qui tente de se relever de la programmation de son anéantissement rejoint celui du début $d u$ siècle qui déclinait la crise d'identité. C'est peut-être là que l'on constate la plus forte communauté d'esprit entre deux époques. Les capitales auxquelles se réfèrent ces écrivains ne sont plus mitteleuropéennes : Tel Aviv, Sao Paulo et New York. La modernité viennoise dont le retentissement fut européen fait place à un déracinement et à une ubiquité qui assure son avenir dans le monde. Le café viennois est le paradigme de la vie citadine, du luxe qui quitte les salons pour descendre dans la rue, symbole de pluralité, d'échanges et d'ouverture à de nouvelles influences, dans son architecture, le café est une conception du monde, un lieu transitoire et éternel ?

La modernité, serait-elle un "projet inachevé $»^{43}$, une réécriture de la tradition, une parole suspendue qui demande à être prolongée? Si elle s'incarne dans le café, revisité par les écrivains d'aujourd'hui, elle représente le topos unique d'un temps où rien ne disparaît, où se mêlent traditions et ruptures, où dialoguent traditions et modernité, non pas dans la linéarité historique mais dans un espace-temps où tout instant s'ouvre sur la réinvention. 


\section{NOTES}

1. Dans son étude de la modernité, Alexis Nouss ouvre la question en affirmant: « (Cela se passe dans un café parisien, évidemment, nommé le Moderne, évidemment.) Dites-moi, c'est moderne en diable de se retrouver dans un café pour parler modernité !», in Alexis Nouss, La modernité, Paris, Granger, 1991, p. 17.

2. Mikhail Bakhtin, «Formes du temps et du chronotope dans le roman 1937-1938 ", in: Esthétique et théorie du roman, Paris, Gallimard, 1978, 488 p.

3. Légende contestée, certainement fausse, mais toujours évoquée, cf. Claudio Magris, Danube, „Les turcs devant Vienne », Paris, „folio », Gallimard, 1986, p. 250.

4. Ibid., p. 248.

5. En souvenir de ceux de 1830 et de 1848 à Paris.

6. Baudelaire in «Le Peintre de la vie moderne ». L'Artiste, homme du monde, homme des foules et enfants, Folio essais, Gallimard, 1992, p. 352.

7. Claudio Magris, op. cit., p. 232.

8. Edmund Wengraf, « Kaffeehaus und Literatur », in Gotthart Wunberg, Wiener Moderne, Literatur, Kunst und Musik, Stuttgart, Reclam, 1981, p. 638

9. Robert Schindel zitiert von Günther Kainsdlstorfer, "Auf Wiederschaun Herr Schindel », in Falter, Wien, Nr. 32, 7. -13. August 1992, pp. 16-18.

10. Cf. Klaus Thiele Dohrmann, Europäische Kaffeehauskultur, Zürich, Artemis \& Winkler, 1997, p. 139.

11. Le cercle Walden : Döblin, Erich Mühsam, Richard Dehmel, Paul Cassirer, cf. ibid.

12. Le café avait été nécessaire à ces gens parce qu'il leur promettait un espace temporaire entre le ghetto et l'émancipation, une sorte de zone de transfert entre talmud et philosophie des Lumières. Cf. Doron Rabinovici, «Lang lebe die Melange », in Format, Wien,12. Oktober 1998, Nr. 2, p.188.

13. À Prague, les écrivains de langue allemande font cercle autour de Gustav Meyrink au Café Continental ; Franz Kafka (1883-1924), lui, retrouve ses compagnons au Café Union.

14. Comme en témoigne S. Zweig dans ses mémoires : «Ainsi nous savions tout ce qui se passait dans le monde, et cela de première main, nous étions renseignés sur tous les livres qui paraissaient, sur toutes les représentations en quelque lieu que ce soit et nous comparions entre elles les critiques de tous les journaux ", in Le Monde d'hier, Souvenirs d'un Européen, Belfond, Paris, 1982 , p. 60.

15. Wiener Moderne, op. cit., pp. 16-19 et p. 643-644.

16. Ibid., p. 292.

17. In Le monde d'hier, op. cit., p. 59.

18. Arthur Schnitzler, Jeunesse viennoise 1862-1889, Autobiographie, Hachette, 1997, p. 134

19. In Elias Canetti, Histoire d'une vie, Le flambeau dans l'oreille, Albin Michel, Paris, 1982, p. 134

20. Jean-Yves Masson, Le lien d'ombre, renoncement et métamorphose, Lagrasse, Verdier, 2006, p. 14-15.

21. Ibid., p. 431.

22. Hofmannsthal, Fremdes Fühlen (Sensation étrange), Ibid., p. 347.

23. Ibid., p. 196.

24. Ibid., p. 12.

25. «Die Frage an das Schicksal» («La question au destin »), in Nouvelle revue de psychanalyse, Gallimard, Paris, $n^{\circ} 30$, p. 171-186.

26. Arthur Schnitzler, Jugend in Wien, op. cit., p. 368.

27. Wiener Moderne, op. cit., p. 494. 
28. Ernst Mach, Die Analyse der Empfindungen und das Verhältnis des Physischen zum Psychischen, Leipzig 1886. Neudruck herausgegeben von Gereon Wolters, Darmstadt 1991.

29. Encyclopédie Universalis entrée Mitteleuropa (Jacques Le Rider).

30. Encyclopédie Universalis entrée « Ernst Mach » (Michel Paty).

31. «Beitrag zur Beurteilung der Lehren Machs von 1908 ».

32. Cf. Béatrice Gonzalés-Vangell, Kaddish et renaissance, Villeneuve d'Asq, Septentrion, 2005, le chapitre « La langue allemande » se trouve pp. 93-112.

33. «Lokalchronik », in Die Nacht der Harlekine, Frankfurt-a-M., Suhrkamp, 1994, p. 80-84.

34. Immernie, Gedichte vom Moos der Neunzighöhlen, Frankfurt-a-M., Suhrkamp, 2000, p. 73.

35. Suche nach M., Frankfurt-a-M., Suhrkamp, 1997, p. 7

36. Ibid., p. 89

37. Gebürtig, Frankfurt-a-M., Suhrkamp, 1992, p. 325, (Le mur de verre, trad. A.-M. Geyer, Paris, Stock, 2005, p. 273).

38. Hugo Bettauer, Die Stadt ohne Juden (1922), Hamburg-Bremen, Achilla, 1996, 194 p.

39. Robert Menasse, Chassés de l'enfer, Verdier, 448 p., trad. de l'allemand (Autriche) par M. Rocher - J. et D. Roche, (Die Vertreibung aus der Hölle, Frankfurt-a-M., Suhrkamp, 2001, 493 p.).

40. Robert Menasse, Die Vertreibung aus der Hölle, p. 320 - Chassés de l'enfer, op. cit., p. 310.

41. Robert Menasse, Sinnliche Gewißheit, Frankfurt-a-M., Suhrkamp, 1996, 329 p.

42. Gebürtig, op. cit., p. 8 - Le Mur de verre, p. 10.

43. Jürgen Habermas, cité in Alexis Nouss, op. cit., p. 168.

\section{RÉSUMÉS}

Le café ou Kaffeehaus, n'est-ce pas la modernité? La présence du café dans la littérature véhicule une certaine idée du monde et du sujet qui l'habite: aussi bien chez Arthur Schnitzler que chez trois écrivains viennois de la fin du xxe siècle: Robert Schindel, Robert Menasse et Doron Rabinovici. La modernité européenne fait place dans la littérature d'aujourd'hui à un déracinement et à une ubiquité qui assure son avenir dans le monde. Cette courte étude posera la question suivante: si l'on retient de la modernité l'idée d'un mouvement inachevé, l'instant d'irruption d'un déchirement de l'identité entre tradition et renouveau, où espace et temps sont indissolubles, ne peut-on pas voir dans le traitement littéraire du lieu café ce que Bakhtin appelait un chronotope?

Ist das Kaffeehaus ein exemplarischer Ort der Bildung der Moderne? Die Darstellung des Kaffeehauses in der Literatur vermittelt eine bestimmte Auffassung der Welt und des in dieser Welt lebenden Subjektes, sowohl bei Arthur Schnitzler, wie auch bei drei Wiener Autoren am Ende des 20. Jahrhunderts: bei Robert Schindel, Robert Menasse und Doron Rabinovici. Auch in der Gegenwartsliteratur ist das Kaffeehaus ein Ort, der den Entwurzelten, den Heimatlosen einen Überlebensraum gibt, wo Tradition und Innovation sich verbinden. Diese kurze Studie stellt die Frage: Wenn sich in modernen Strukturen von Kaffeehaus-Erzählungen Tradition und Innovation verbinden, ist es dann nicht möglich, in der literarischen Behandlung des Ortes Kaffeehaus das zu sehen, was Bakthin Chronotopus nannte? 
INDEX

Mots-clés : Kaffeehaus, modernité 